\title{
Genetic generalized and focal epilepsy prevalence in the North American SUDEP Registry
}

Chloe Verducci, BA, Daniel Friedman, MD, MSc, Elizabeth Donner, MD, MSc, FRCPC, and Orrin Devinsky, MD

Neurology ${ }^{\circledR}$ 2020;94:e1757-1763. doi:10.1212/WNL.0000000000009295

\section{Abstract}

\section{Objective}

To assess relative rates and clinical features of patients with genetic generalized epilepsy (GGE), focal epilepsy (FE), and developmental encephalopathic epilepsy (DEE) in the North American SUDEP Registry (NASR).

\section{Methods}

We identified all adjudicated definite, definite plus, and probable sudden unexpected death in epilepsy (SUDEP) cases $(n=262)$ and determined epilepsy type (GGE, FE, or DEE) from medical record review including history, imaging and EEG results, genetics, and next-of-kin interviews.

\section{Results}

Of the 262 SUDEP cases, 41 occurred in GGE, 95 in FE, 24 in DEE, and 102 were unclassifiable. GGE cases comprised $26 \%$ of NASR cases with an epilepsy syndrome diagnosis. The relative frequency of FE:GGE was slightly lower (2.3:1) than in population cohorts (2.1-6: 1). Compared to patients with FE, patients with GGE had similar (1) ages at death and epilepsy onset and rates of (2) terminal and historical antiseizure medication adherence; (3) abnormal cardiac pathology; (4) illicit drug/alcohol use histories; and (5) sleep state when SUDEP occurred.

\section{Conclusions}

GGE cases were relatively overrepresented in NASR. Because GGEs are less often treatmentresistant than FE or DEE, seizure type rather than frequency may be critical. Many people with GGE predominantly have generalized tonic-clonic seizures (GTCS) when they have uncontrolled or breakthrough seizures, whereas patients with FE more commonly experience milder seizures. Future mechanistic SUDEP studies should assess primary and focal-to-bilateral GTCS to identify potential differences in postictal autonomic and arousal disorders and to determine the differential role that lifestyle factors have on breakthrough seizures and seizure types in GGE vs FE to effectively target SUDEP mechanisms and prevention.

\author{
Correspondence \\ Dr. Devinsky \\ od4@nyu.edu
}

\section{RELATED ARTICLE}

Editorial

Patients with generalized epilepsy are also at risk for SUDEP

Page 689 


\section{Glossary}

ASM = antiseizure medication; DEE = developmental encephalopathic epilepsy; $\mathbf{E M U}=$ epilepsy monitoring unit; $\mathbf{F E}=$ focal epilepsy; GGE = genetic generalized epilepsy; GTCS = generalized tonic-clonic seizures; IQR = interquartile range; JME = juvenile myoclonic epilepsy; NASR = North American SUDEP Registry; NOK = next of kin; PGES = postictal generalized EEG suppression; SUDEP = sudden unexpected death in epilepsy.

Genetic generalized epilepsies (GGEs) are common in children and adults, comprising $23 \%-35 \%$ of all epilepsy syndromes. ${ }^{1,2}$ GGEs are usually treatment-responsive, although $15 \%-18 \%$ of patients experience ongoing seizures despite adherence with appropriate antiseizure medications (ASMs). ${ }^{3-6}$ However, GGEs account for $6 \%-8 \%$ of drugresistant epilepsies. ${ }^{7}$

The strongest risk factors for sudden unexpected death in epilepsy (SUDEP) in epidemiologic, case-control, and epilepsy monitoring unit (EMU) studies are generalized tonic-clonic seizures (GTCS) that are recent, nocturnal, or frequent, as well as nonadherence to ASMs and treatment resistance, ${ }^{8}$ all reflecting poor seizure control. ${ }^{9}$ Proposed SUDEP biomarkers, including peri-ictal central apnea and subsequent hypoxemia, ${ }^{10}$ are best defined in focal-onset seizures. ${ }^{11}$ However, postconvulsive central apnea, most directly relevant to SUDEP mechanisms in the MORTEMUS series, ${ }^{12}$ can occur after focal or generalized onset GTCS. $^{13}$

Most SUDEP series come from epidemiologic studies based on population-based data, or consecutive medical examiner cases in which detailed seizure histories are limited, and less frequently tertiary care epilepsy treatment centers. Furthermore, most pathomechanistic SUDEP studies focus on focal-to-bilateral GTCS; we lack data on primary GTCS. ${ }^{14-16}$ Patients with focal epilepsy (FE) are considered higher risk for SUDEP than those with GGE, based on studies of post-focal onset GTCS hypoxemia and respiratory distress (highest in temporal lobe epilepsies), and due to high relative rates of seizure intractability. ${ }^{7,15,16}$ Other series focus on patients at high SUDEP risk with developmental encephalopathic epilepsies (DEEs), such as Dravet syndrome ${ }^{17}$ or dup $15 q$ syndrome. ${ }^{18}$

We assessed the frequency of GGE in a consecutive series of 262 SUDEP cases enrolled in the North American SUDEP Registry (NASR).

\section{Methods}

\section{Standard protocol approvals, registrations, and patient consents}

From October 2011, NASR enrolled 526 cases of suspected sudden death among people with epilepsy, deceased epilepsy controls, living people with epilepsy, and immediate family members of epilepsy decedents. SUDEP adjudication was completed by 2 epileptologists with expertise in epilepsyrelated mortality (O.D. and D.F.), with input from a third expert in cases of disagreement (E.D.). ${ }^{19} \mathrm{~A}$ total of 262 cases were adjudicated as definite, definite plus, or probable SUDEP using Nashef et al. ${ }^{20}$ criteria.

Cases were classified by epilepsy etiology based on 2 epileptologists' adjudications of seizure type and full medical record review for any relevant history that would reasonably explain epilepsy ${ }^{21}$ (for example, epilepsy of clear structural, infectious, or immune origin). Generalized epilepsies that had no likely cause and cases that were diagnosed by a previous treating neurologist/epileptologist as "cryptogenic" or "idiopathic" in origin were classified as GGE. In this study, treatment resistance is defined as persistent seizures despite adequate trials of at least 2 appropriate antiseizure medications.

Interview content included next-of-kin (NOK) descriptions of seizure phenotypes, epilepsy treatment/ASM adherence, family history of epilepsy, and comorbid health conditions. These histories were corroborated, when possible, with medical records obtained from previously treating physicians, neurologists, and investigative/autopsy reports. In the event of a discrepancy-most frequently regarding terminal and historical ASM adherence-toxicology and other laboratory reports were considered to be most accurate.

\section{Standard protocol approvals, registrations, and patient consents}

Full NASR intake and interview methodology was reported, ${ }^{22}$ and all NOK of participants provided informed consent to participate in this study. This study was approved by the New York University Langone Medical Center Institutional review board.

\section{Statistical analysis}

A Kruskal-Wallis test was performed to compare age at death and age at epilepsy onset among the GGE, FE, and DEE cohorts, with a Dunn post hoc test. Chi-square tests were performed to determine differences in medication compliance, both historically and for terminal dose, alcohol use, and presence/absence of witnessed terminal seizure. All statistics were performed using SPSS version 23 (Armonk, NY).

\section{Data availability}

Deidentified data will be available upon request to any qualified researcher. 


\section{Results}

\section{Participants}

Of the 262 cases adjudicated as definite, definite plus, or probable SUDEP, 160 had sufficient information in medical records and interview transcripts with NOK to adjudicate epilepsy syndrome as GGE, FE, or DEE using the International League Against Epilepsy 2017 criteria. $^{21}$ Of these 160 cases, 41 were GGE, 95 FE, and 24 DEE. Three additional cases were excluded as structural generalized epilepsy, originating from hypoxic-ischemic encephalopathy, significant head trauma with generalized seizures immediately following impact, or tuberous sclerosis; 1 Unverricht-Lundborg disease case was excluded. The remaining 99 cases had insufficient information to determine if seizures were focal or generalized in onset $(n=30)$, insufficient medical history to determine whether or not there was a structural or symptomatic etiology of epilepsy $(n=47)$, or both $(n=22)$. Scripted interview was conducted in $80 \%$ of cases: $76 \%$ of FE cases, $92 \%$ of DDE cases, and $83 \%$ of GGE cases.

EEGs were collected for $80 \%$ of GGE cases (33/41). Four of these cases had recorded seizures to corroborate diagnosis of primary generalized epilepsy. Recorded seizures included tonic-clonic, clonic, typical absence, atypical absence, and tonic, with 1 patient experiencing generalized seizures not otherwise specified. In the remaining 8 cases without EEGs, either EEG was performed previously but these records could not be obtained, or the decedent had no history of EEG. For the GGE cases without obtainable EEG, diagnosis was made by epileptologist review of history (e.g., age at onset, family history of epilepsy), seizure semiology, and absence of competing structural or metabolic causes of epilepsy. Interictal activity was used in a majority of cases with EEG (86\%) to corroborate GGE diagnosis. MRI reports were available in $61 \%$ of GGE cases, and imaging was normal in most (76\%), with only incidental findings in the rest (i.e., isolated venous anomaly, arachnoid cysts).

Three of the 41 GGE cases had genetic testing; none of these identified known pathogenic variants. Among the $24 \mathrm{DEE}$ cases, 92\% had genetic studies: 13 had SCN1A variants (some pathogenic, all corroborated as DEEs by clinical evidence of Dravet syndrome), 7 had isodicentric 15th chromosome (idic/dup15q syndrome), and one had no known pathogenic gene variants (figure e-1 and table e-1, doi:10.5061/dryad. $9 \mathrm{kd} 51 \mathrm{c} 5 \mathrm{c} 6)$. Eleven of the 95 (12\%) FE cases had genetic testing; the only positive findings were 1 case with NOTCH3 mutation (strong family history) and 1 with XYY karyotype.

Among GGE cases, 10 (24\%) patients had a first-degree relative with epilepsy, including 2 siblings who also died of SUDEP. An additional 13 GGE decedents had non-firstdegree relatives with epilepsy, including a first cousin who died of SUDEP. Type of epilepsy in family members was poorly recalled except in 1 case, a twin with unclassified tonicclonic seizures who died of definite SUDEP and is also enrolled in NASR. Four (10\%) patients with GGE had juvenile myoclonic epilepsy (JME), 3 patients had febrile seizures plus (7\%), and 1 each (2\%) had benign familial infantile epilepsy, epilepsy with GTCS on awakening, and Jeavons syndrome. The rest had GGE without syndromic diagnosis.

The median age at death for people with exclusively GGE ( $\mathrm{n}=$ $41)$ was 26 years (interquartile range $[I Q R], 20-34$ years), similar to the FE group $(\mathrm{n}=95)$ (26 years, IQR 20-38 years), but both were older than the DEE group $(n=24,14$ years [IQR 8-21 years]; $p<0.001$ for GGE or FE vs DEE). The median age at epilepsy onset was 13 years for GGE, 12 years for FE, and 0.7 years for DEE $(p<0.001)$. Patients with DEEs had significantly higher reported rates of ASM adherence (88\%) compared to those with FEs (43\%) or GGEs (54\%) ( $p$ $<0.01)$. The mean number of ASMs taken at time of death were 2 (SD 1) for the GGE group, 3 (SD 2) for the DEE group, and 2 (SD 1) for the FE group. Sixty-eight percent (28/41) of GGE, $100 \%(24 / 24)$ of DEE, and 66\% (63/95) of FE cases were medically refractory, defined as failing at least 2 ASMs. The last dose of ASMs before death was taken near the scheduled time by $47 / 106$ (44\%) of all patients with available information (i.e., they missed their last 1 or more doses), with similar rates of reported "final" adherence in GGE (37\%) and FE (43\%), but greater terminal ASM adherence in DEE $(58 \%)$ cases $(p<0.05)$.

Figure shows the estimated lifetime GTCS frequency distributions for each group.

History of non-seizure-related cardiac arrhythmia was diagnosed in 2 GGE cases, 9 FE cases, and no DEE cases; ictal arrhythmia was noted in 3 GGE cases ( 1 bradycardia, 1 firstdegree arteriovenous block, 1 unknown), $5 \mathrm{FE}$ cases (1 bradycardia, 1 with Wolf-Parkinson-White syndrome, and 3 unknown), and 3 DEE cases (1 ventricular tachycardia, 1 ventricular fibrillation, 1 unknown). Formal EKG reports were obtained for $10 \mathrm{GGE}$ cases ( 6 normal), $18 \mathrm{FE}$ cases ( 8 normal), and 2 DEE cases (1 normal). Most common EKG findings for the GGE group included $\mathrm{T}$-wave inversion, $\mathrm{ST}$ elevation, sinus tachycardia, and bradycardia. The FE group had a similar EKG profile. Cardiac comorbidities that were not the cause of death but could have contributed in some cases were present in in $15 \%$ of patients with GGE, $16 \%$ of patients with $\mathrm{FE}$, and $8 \%$ of the DEE group. Gross and microscopic cardiac pathologic findings were similar for GGE and FE, while autopsies were infrequent (9/24) in DEE cases. Cardiac and pulmonary autopsy findings for each group are listed in table 1.

A history of current alcohol use among patients $\geq 21$ years old was identified in $28 \%$ of GGE cases, $27 \%$ of FE cases, and $0 \%$ of the DEE group $(p<0.05)$. Non-alcohol substance abuse occurred in $22 \%$ of GGE cases, $19 \%$ of FE cases, and $0 \%$ of DEE cases; of these recreational drug users, cannabis was used in $89 \%-90 \%$. Summary data for each group are shown in table 2 . 
Figure Distribution of estimated lifetime generalized tonic-clonic seizure counts in individuals with genetic generalized epilepsy (GGE), developmental encephalopathic epilepsy (DEE), and focal epilepsy (FE)

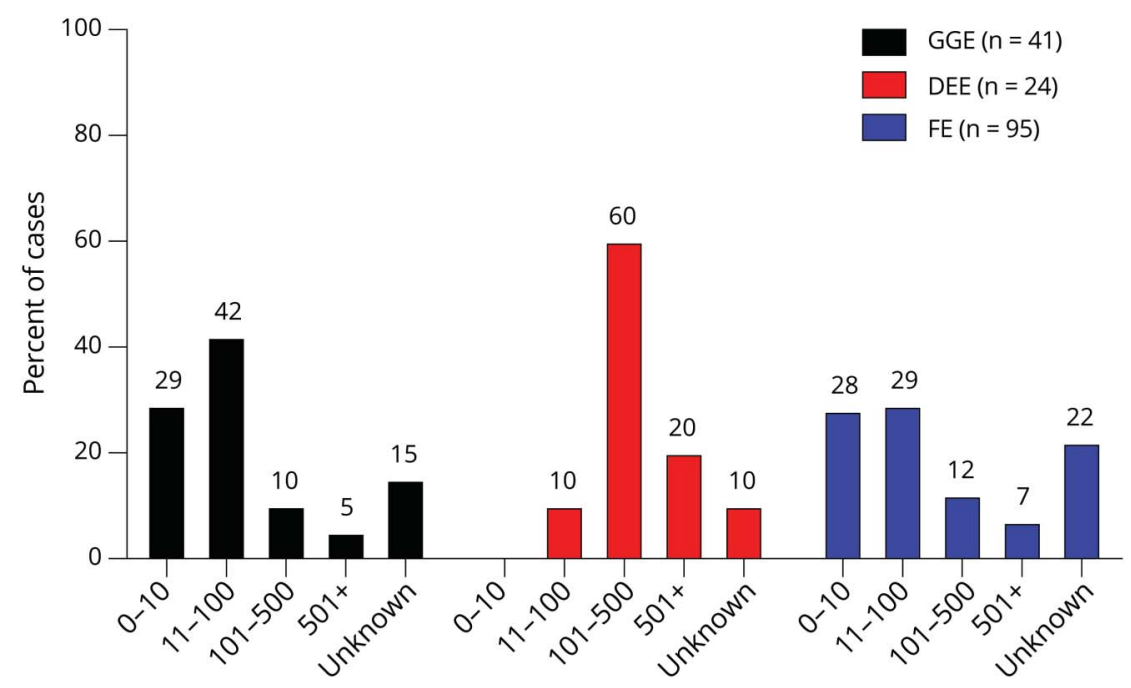

Death was witnessed in $10 \%$ of GGE cases, $17 \%$ of DEE cases, and $3 \%$ of FE cases. A seizure "around time of death" was witnessed by interviewees in $12 \%$ of all GGEs, $21 \%$ of DEEs, and $8 \%$ of FEs, most often immediately before or during sleep $(p>0.05)$. In most cases where no seizure was witnessed immediately preceding death but there was evidence a seizure had occurred, common death scene observations included unusual positioning of the body (66\% GGE, 35\% DEE, $45 \%$ $\mathrm{FE}$ ), incontinence (29\% GGE, 6\% DEE, 21\% FE), and blood on the pillow around the mouth (33\% GGE, 0\% DEE, 23\% FE) $(n=24 / 41$ GGE, $n=17 / 24$ DEE, $n=56 / 95 \mathrm{FE})$. Fortysix percent of GGE, $76 \%$ of DEE, and $63 \%$ of FE decedents were sleeping at time of death, with the most common location of death in every group being at home, in bed.

\section{Discussion}

Patients with GGE comprised 26\% of SUDEP cases in NASR with an epilepsy syndrome diagnosis. The relative frequency of FE and GGE (2.3:1) in NASR was within the lower range of prevalence in the general epilepsy population $\left(2.1^{23}-6: 1^{24}\right)$, although older studies frequently underestimated the $\mathrm{FE}$ prevalence due to insufficient diagnostic information to distinguish focal- from generalized-onset tonic-clonic seizures. ${ }^{25}$ Our findings-that GGEs account for over $1 / 4$ of SUDEPs—-suggest that these epilepsies, considered treatmentresponsive with infrequent seizures, are associated with sudden unexpected death. Compared to GGE, FE is $>2$-fold more prevalent and 3- to 11-fold more likely to be uncontrolled despite appropriately selected ASMs. We expected that the frequency of SUDEP in FE would be much higher (e.g., $>6-22 x$ ) than in GGE. ${ }^{26,27}$ Individuals with GGE, compared to other epilepsies, are overrepresented in this series, if accounting for the relative frequency and rates of drug resistance. Thus,
SUDEP risk appears to be driven not by the epilepsy syndrome, but by the type of seizures that tend to be drug-resistant or breakthrough after missed medication, sleep deprivation, alcohol withdrawal, stress, or other factors. Some epilepsies that are usually well-controlled with ASM, including epilepsy with tonic-clonic seizures on awakening and JME, are characterized by predominantly nocturnal or sleep-related seizures, ${ }^{28}$ which may contribute to the relatively high frequency of GGE cases in NASR, as nocturnal seizures may be an independent risk factor for SUDEP. ${ }^{29}$ Further, treatment resistance rates were similarly high in both the GGE and FE cases in NASR.

Although more GGEs occurred in our cohort than predicted based on epidemiologic prevalence studies, ${ }^{23,27}$ it is uncertain if patients with GGE are at increased risk for SUDEP compared to patients with $\mathrm{FE}$, independent from the observation that people with GGEs are more likely to have more frequently refractory tonic-clonic seizures than people with $\mathrm{FE}$ (although FEs are more frequently treatment-resistant than $\mathrm{GGEs}^{3-7}$ the seizures that persist are usually [>85\%] nonconvulsive and therefore less likely to cause SUDEP). ${ }^{30,31} \mathrm{We}$ also noted a large number of patients with SCN1A variants and chromosome $15 \mathrm{q}$ duplications within the DEE cohort. This is likely a result of referral bias, as NASR frequently collaborates with lay advocacy organizations for both syndromes (Dup15q Alliance and the Dravet Foundation).

A multicenter prospective study of seizures recorded in the EMU found $86 \%$ of patients with focal-onset GTCS experience severe peri-ictal or postictal hypoxemia. ${ }^{15}$ Postictal hypoxemia during the focal phase of secondary GTCS is highly correlated with low oxygen saturation recovery and postictal generalized EEG suppression (PGES). However, it is unknown whether postictal cardiopulmonary dysfunction varies between primary and focal-to-bilateral GTCS, because 
Table 1 Cardiac and pulmonary gross and histologic autopsy findings by epilepsy type

\begin{tabular}{|c|c|c|c|c|}
\hline & \multicolumn{2}{|l|}{ Cardiac } & \multicolumn{2}{|l|}{ Pulmonary } \\
\hline & Gross & Histologic & Gross & Histologic \\
\hline \multicolumn{5}{|l|}{ GGE } \\
\hline Unremarkable & 17 & 8 & 6 & 6 \\
\hline \multirow[t]{4}{*}{ Abnormality } & 12 & 17 & 24 & 19 \\
\hline & 6 Atherosclerosis & 5 Fibrosis & 12 Edema & 16 Congestion \\
\hline & 5 Gross cardiomegaly & 4 Hypertrophy & 8 Congestion & 8 Edema \\
\hline & 2 Atrial enlargement & 3 Congestion & 5 Froth/foam & 2 Macrophages \\
\hline Not examined & 12 & 16 & 13 & 17 \\
\hline \multicolumn{5}{|l|}{ FE } \\
\hline Unremarkable & 33 & 25 & 15 & 11 \\
\hline \multirow[t]{4}{*}{ Abnormality } & 25 & 21 & 43 & 37 \\
\hline & 11 Atherosclerosis & 11 Fibrosis & 28 Congestion & 29 Congestion \\
\hline & 9 Hypertrophy & 10 Hypertrophy & 19 Edema & 25 Edema \\
\hline & 5 Gross cardiomegaly & 2 Congestion & 10 Froth/foam & 4 Macrophages \\
\hline Not examined & 36 & 48 & 36 & 46 \\
\hline \multicolumn{5}{|l|}{ DEE } \\
\hline Unremarkable & 7 & 7 & 3 & 2 \\
\hline \multirow[t]{4}{*}{ Abnormality } & 2 & 1 & 6 & 5 \\
\hline & 1 Hypertrophy & 1 Congestion & 5 Congestion & 4 Congestion \\
\hline & 1 Dilated atria and ventricles & & 5 Edema & 2 Edema \\
\hline & & & 2 Froth/foam & 1 Macrophages \\
\hline Not examined & 15 & 16 & 15 & 17 \\
\hline
\end{tabular}

Abbreviations: DEE = developmental encephalopathic epilepsy; FE = focal epilepsy; GGE = genetic generalized epilepsy.

most cases admitted and provoked in EMUs are for surgical evaluation, and since patients with GGE are not eligible, there is referral bias. ${ }^{15}$

PGES and respiratory dysfunction in nocturnal seizures last significantly longer, result in longer recovery time, and carry risk of more severe desaturation than seizures during wakefulness. ${ }^{14,32}$ However, only $6 \%$ of one cohort had primary GTCS—-the vast majority had focal onset GTCS, so no comparison between types of GTCS could be made. ${ }^{14}$ A similar study exploring the effect of potential high-risk cardiac arrhythmias on duration of ictal hypoxemia only included EMU data for a single primary GTCS case; the rest were focal onset GTCS. ${ }^{16}$

Despite multiple studies showing that focal-onset GTCS exhibit prolonged nocturnal PGES, respiratory distress, ictal hypoxemia, and subsequent prolonged postictal immobility, and the concept that people with GGE are at a lower risk of SUDEP, our data suggest that people with GGE also bear significant SUDEP risk. Population-based studies are needed to ascertain the true relative frequencies of SUDEP among GGE and FE. The common belief that people with GGE have lower SUDEP risk than people with FE is based on literature derived primarily from EMU studies, in which the majority of patients $(>90 \%)$ have FEs. Studies to compare potential SUDEP markers such as PGES, ictal hypoxemia, and subsequent prolonged postictal immobility between focal and primary GTCS are needed. Video-EEG analyses of nocturnal GTCS should include cases with varied GTCS localizations that accurately account for the heterogeneity of SUDEP cases, since we found slight overrepresentation among GGEs given their prevalence in the drug-resistant population. ${ }^{23,27}$

SUDEP is a continuous risk in people with epilepsy every year after onset of epilepsy-for this reason, one might expect that patients with GGE syndromes with predominant onset in childhood (generalized epilepsy with febrile seizures plus, ${ }^{33} \mathrm{JME}^{34}$ ) are at increased SUDEP risk 
Table 2 Profiles of genetic generalized epilepsy (GGE), focal epilepsy (FE), and developmental and epileptic encephalopathy (DEE) groups in the North American SUDEP Registry

\begin{tabular}{llll}
\hline & GGE $(\mathbf{n}=\mathbf{4 1})$ & FE $(\mathbf{n}=\mathbf{9 5})$ & DEE $(\mathbf{n}=\mathbf{2 4})$ \\
\hline Median age at death, $\mathbf{y}$ & 26 & 26 & 14 \\
\hline Median age at onset, $\mathbf{y}$ & 13 & 12 & 0.7 \\
\hline ASM compliant & $54 \%$ & $43 \%$ & $88 \%$ \\
\hline Drug use, current & $15 \%$ & $12 \%$ & $0 \%$ \\
\hline Alcohol use, current & $28 \%$ & $27 \%$ & $0 \%$ \\
\hline Cardiac condition (Other) & $15 \%$ & $16 \%$ & $8 \%$ \\
\hline Syncope & $12 \%$ & $8 \%$ & $10 \%$ \\
\hline Arrhythmia & $5 \%$ & $10 \%$ & $0 \%$ \\
\hline Took last dose? & $37 \%$ & $43 \%$ & $58 \%$ \\
\hline
\end{tabular}

Abbreviations: ASM = antiseizure medication; SUDEP = sudden unexpected death in epilepsy.

because they sustain more life-years with epilepsy than those with focal etiologies. However, in NASR, ages at GGE and FE epilepsy onset were very similar (13 and 12 years, respectively), suggesting this did not contribute to our high frequency of GGE cases.

Several factors could explain the unexpectedly high rate of patients with GGE among our SUDEP cohort. A key risk factor for SUDEP in GGE is that many adolescents and adults with GGE have GTCS as the predominant seizure type when they have uncontrolled seizures or breakthrough seizures. In contrast, for patients with $\mathrm{FE}$, milder seizures are most common. In investigative death reporting, slightly more decedents with GGE were discovered with evidence of an unwitnessed recent GTCS, based on a tongue bite or blood on pillow, urinary incontinence, and unusual body positioning (i.e., falling out of bed). This, in conjunction with the many varied groups and presentations of SUDEP, ${ }^{22}$ may indicate that each epilepsy subgroup has different risk factors or ultimate SUDEP mechanism.

GGEs are associated with deficits in memory and executive function. ${ }^{35-37}$ These problems may be nonprogressive or progress slowly. ASM adherence is improved when the burden of medication responsibility is placed upon caregivers. ${ }^{38}$ Patients with GGEs such as JME have impairments in executive functions including mental flexibility and concept formation, judgment, ability to inhibit learned behavioral responses that are maladaptive in the current environment, organization and adaptive behavior, and self-initiation of actions without environmental stimuli. ${ }^{39-41}$ Patients with JME are also more likely to engage in risk-taking behaviors than other epilepsy groups. ${ }^{42}$ The impulsive and "environmentally driven" risky behaviors fostered by frontal dysfunction may make patients with JME more likely to increase seizure risk due to sleep deprivation, alcohol intoxication and withdrawal, substance abuse, and nonadherence. Breakthrough seizures due to provocative factors may not be classified as "treatment-resistant" since seizures resulted from lifestyle factors, not ASM failure. Although executive dysfunction is associated with ASM nonadherence, ${ }^{43}$ our patients with GGE who experienced SUDEP had slightly higher rates of historical ASM adherence than patients with FE, and nearly identical rates of terminal ASM adherence. However, this difference was not significant, and data relied primarily on NOK recall, as records infrequently document ASM adherence, even at tertiary care epilepsy centers. ASM nonadherence may be more dangerous in GGE than FE, in which the resultant GTCS vs nonconvulsive seizure may be more likely to cause SUDEP.

Prospective studies are needed to assess the effects of GTCS on autonomic regulation and arousal, and to determine the differential role that lifestyle factors have on breakthrough seizures and seizure types in GGE vs FE, to effectively target SUDEP mechanisms and prevention.

\section{Study funding}

This study was funded by Finding a Cure for Epilepsy and Seizures, Lundbeck, and American Epilepsy Society.

\section{Disclosure}

C. Verducci reports no relevant disclosures. D. Friedman receives salary support for consulting and clinical trial related activities performed on behalf of The Epilepsy Study Consortium, a nonprofit organization, and receives no personal income for these activities; NYU receives a fixed amount from the Epilepsy Study Consortium towards Dr. Friedman's salary; within the past year, The Epilepsy Study Consortium received payments for research services performed by Dr. Friedman from Adamas, Biogen, CuroNZ, Engage Pharmaceuticals, Eisai, GW Pharmaceuticals, Pfizer, Takeda, and Zynerba; D. Friedman has served as a paid consultant for Eisai and Penumbra; has received honorarium from Neuropace, Inc.; receives research support from Empatica, Epitel, UCB, Inc., and Neuropace for work unrelated to this study; serves on the scientific advisory board for Receptor Life Sciences; and holds equity interests in Neuroview Technology. E. Donner has received honoraria from Eisai and UCB and receives research funding from the Canadian Institutes of Health Research and Ontario Brain Institute. O. Devinsky is the Principal Investigator for the North American SUDEP Registry, the SUDC Registry \& Research Collaborative, and serves on the Executive Committee of the Epilepsy Foundation Center for SUDEP Research; is funded by the National Institute of Neurological Disorders and Stroke Center for SUDEP Research; has equity interests in Empatica, Receptor Life Sciences, Tilray/Privateer Holdings, Papa \& Barkeley, RETTCO, Tevard, and Q-State Biosciences; and has consulted for GW Pharmaceuticals and Cavion. Go to Neurology.org/N for full disclosures. 


\section{Publication history}

Received by Neurology February 28, 2019. Accepted in final form November 18, 2019.

\section{Appendix Authors}

\begin{tabular}{lll}
\hline Name & Location & Contribution \\
\hline $\begin{array}{l}\text { Chloe } \\
\text { Verducci, BA }\end{array}$ & $\begin{array}{l}\text { Comprehensive Epilepsy } \\
\text { Center, New York } \\
\text { University School of } \\
\text { Medicine }\end{array}$ & $\begin{array}{l}\text { Drafting and revision, data } \\
\text { collection, statistical } \\
\text { analysis }\end{array}$ \\
\hline $\begin{array}{l}\text { Daniel } \\
\text { Friedman, } \\
\text { MD, MSc }\end{array}$ & $\begin{array}{l}\text { Comprehensive Epilepsy } \\
\text { Center, New York }\end{array}$ & $\begin{array}{l}\text { Designed and } \\
\text { conceptualized study, } \\
\text { drafting and revision, data } \\
\text { collection, statistical analysis }\end{array}$ \\
\hline $\begin{array}{l}\text { Elizabeth } \\
\text { Donner, MD, } \\
\text { MSc, FRCPC }\end{array}$ & $\begin{array}{l}\text { The Hospital for Sick } \\
\text { Children, Division of } \\
\text { Neurology, University of }\end{array}$ & $\begin{array}{l}\text { Designed and } \\
\text { conceptualized study, } \\
\text { drafting and revision }\end{array}$ \\
\hline $\begin{array}{l}\text { Orronto, Canada } \\
\text { Devinsky, } \\
\text { MD }\end{array}$ & $\begin{array}{l}\text { Comprehensive Epilepsy } \\
\text { Center, New York }\end{array}$ & $\begin{array}{l}\text { Designed and } \\
\text { conceptualized study, data } \\
\text { collection, drafting and } \\
\text { revision }\end{array}$ \\
\hline & Mniversity School of & \\
\hline
\end{tabular}

\section{References}

1. Modalsli Aaberg K, Surén P, Lund Søraas C, et al. Seizures, syndromes, and etiologies in childhood epilepsy: the International League Against Epilepsy 1981, 1989, and 2017 classifications used in a population-based cohort. Epilepsia 2017;58:1880-1891.

2. Wirrell E, Grossardt B, Wong-Kisiel L, Nickels K. Incidence and classification of newonset epilepsy and epilepsy syndromes in children in Olmsted County, Minnesota from 1980 to 2004: a population-based study. Epilepsy Res 2011;95:110-118.

3. Semah F, Picot MC, Adam C, et al. Is the underlying cause of epilepsy a major prognostic factor for recurrence? Neurology 1998;51:1256-1262.

4. Berg A, Shinnar S, Levy S, et al. Two-year remission and subsequent relapse in children with newly diagnosed epilepsy. Epilepsia 2002;42:1553-1562.

5. Mohanraj R, Brodie M. Outcomes of newly diagnosed idiopathic generalized epilepsy syndromes in a non-pediatric setting. Acta Neurol Scand 2007;115:204-208.

6. Szaflarski J, Lindsell C, Zakaria T, Banks C, Privitera M. Seizure control in patients with idiopathic generalized epilepsies: EEG determinants of medication response. Epilepsy Behav 2010;17:525-530.

7. Alexandre V Jr, Capovilla G, Fattore C, et al. Characteristics of a large population of patients with refractory epilepsy attending tertiary referral centers in Italy. Epilepsia 2010;51:921-925.

8. Devinsky O, Hesdorffer D, Thurman D, Lhatoo S, Richerson G. Sudden unexpected death in epilepsy: epidemiology, mechanisms, and prevention. Lancet Neurol 2016;15:1075-1088.

9. DeGiorgio C, Markovic D, Mazumder R, Moseley B. Ranking the leading risk factors for sudden unexpected death in epilepsy. Front Neurol 2017;8:1-6.

10. So E, Sam M, Lagerlund T. Postictal central apnea as a cause of SUDEP: evidence from near-SUDEP incident. Epilepsia 2005;41:1494-1497.

11. Lacuey N, Zonjy B, Hampson J, et al. The incidence and significance of periictal apnea in epileptic seizures. Epilepsia 2018;59:573-582.

12. Ryvlin P, Nashef L, Lhatoo S, et al. Incidence and mechanisms of cardiorespiratory arrests in epilepsy monitoring units (MORTEMUS): a retrospective study. Lancet Neurol 2013;12:966-977.

13. Vilella L, Lacuey N, Hampson J, et al. Postconvulsive central apnea as a biomarker for sudden unexpected death in epilepsy (SUDEP). Neurology 2018;92:e1-e12.

14. Peng W, Danison J, Seyal M. Postictal generalized EEG suppression and respiratory dysfunction following generalized tonic-clonic seizures in sleep and wakefulness. Epilepsia 2017;58:1409-1414.

15. Rheims S, Alvarez B, Alexandre V, et al. Hypoxemia following generalized convulsive seizures: risk factors and effect of oxygen therapy. Neurology 2019;92:e183-e193.

16. Park K, Sharma G, Kennedy J, Seyal M. Potentially high-risk cardiac arrhythmias with focal to bilateral tonic-clonic seizures and generalized tonic-clonic seizures are associated with the duration of periictal hypoxemia. Epilepsia 2017;58:2164-2171.
17. Cooper M, Mcintosh A, Crompton D, et al. Mortality in Dravet syndrome. Epilepsy Res 2016;128:43-47.

18. Friedman D, Thaler A, Thaler J, et al. Mortality in isodicentric chromosome 15 syndrome: the role of SUDEP. Epilepsy Behav 2016;61:1-5.

19. Devinsky O, Bundock E, Hesdorffer D, et al. Resolving ambiguities in SUDEP classification. Epilepsia 2018;59:1220-1233.

20. Nashef L, So E, Ryvlin P, Tomson T. Unifying the definitions of sudden unexpected death in epilepsy. Epilepsia 2011;53:227-233.

21. Schefer I, Berkovic S, Capovilla G, et al. ILAE classification of the epilepsies: position paper of the ILAE commission for classification and terminology. Epilepsia 2017;58: $512-521$.

22. Verducci C, Hussain F, Donner E, et al. SUDEP in the North American SUDEP Registry: the full spectrum of epilepsies. Neurology 2019;93:e227-e236.

23. Neligan A, Sander J, The Incidence and Prevalence of Epilepsy, 2005 [online]. Available at: e-epilepsy.org.uk/. Accessed February 1, 2019

24. Manford M, Hart Y, Sander J, Shorvon S. The National General Practice Study of Epilepsy: the syndromic classification of the International League Against Epilepsy applied to epilepsy in a general population. Arch Neurol 1992;49: 801-808.

25. Kirton A, Darwish H, Wirrell E. Unique clinical phenomenology can help distinguish primary from secondary generalized seizures in children. J Child Neurol 2004;19. $265-270$

26. Choi H, Hayat M, Zhang R, et al. Drug-resistant epilepsy in adults: outcome trajectories after failure of two medications. Epilepsia 2016;57:1152-1160.

27. Conte F, Legros B, Van Paesschen W, Avbersek A, Muglia P, Depondt C. Longterm seizure outcomes in patients with drug-resistant epilepsy. Seizure 2018; 62:74-78.

28. Schmitt B. Sleep and epilepsy syndromes. Neuropediatrics 2015;46:171-180.

29. Lamberts R, Thijs R, Laffan A, Langan Y, Sander J. Sudden unexpected death in epilepsy: people with nocturnal seizures may be at highest risk. Epilepsia 2011;53: 253-257.

30. Abarrategui B, García-García ME, Toledano R, Parejo-Carbonell B, Gil-Nagel A, García-Morales I. Lacosamide for refractory generalized tonic-clonic seizures of nonfocal origin and clinical practice: a clinical and VEEG study. Epilepsy Behav Case Rep 2017;8:63-65.

31. Golyala A, Kwan P. Drug development for refractory epilepsy: the past 25 years and beyond. Seizure 2017;44:147-156

32. Latreille V, Abdennadher M, Dworetzky B, et al. Nocturnal seizures are associated with more severe hypoxemia and increased risk of postictal generalized EEG suppression. Epilepsia 2017;58:e127-e131.

33. Lee YJ, Hwang SK, Kwon S. The clinical spectrum of benign epilepsy with centrotemporal spikes: a challenge in categorization and predictability. J Epilepsy Res 2017, $7: 1-6$

34. Syvertsen M, Hellum MK, Hansen G, et al. Prevalence of juvenile myoclonic epilepsy in people $<30$ years of age: a population-based study in Norway. Epilepsia 2016;58: $105-112$

35. Pavone P, Bianchini R, Trifiletti RR, Incorpora G, Pavone A, Parano E. Neuropsychological assessment in children with absence epilepsy. Neurology 2001;56: 1047-1051

36. Parrish J, Jones J, Seth R, Hermann B, Seidenberg M. Executive functioning in childhood epilepsy: parent-report and cognitive assessment. Dev Med Child Neurol 2007;49:412-416

37. Hoie B, Sommerfelt K, Waaler PE, Alsaker FD, Skeidsvoll H, Mykletun A. The combined burden of cognitive, executive function, and psychosocial problems in children with epilepsy: a population-based study. Dev Med Child Neurol 2008;50: 530-536.

38. Holbein C, Smith A, Peugh J, Modi A. Allocation of treatment responsibility in adolescents with epilepsy: associations with cognitive skills and medication adherence. J Pediatr Psychol 2019;44:72-83.

39. Devinsky O, Gershengorn J, Brown E, Perrine K, Vazquez B, Luciano D. Frontal functions in juvenile myoclonic epilepsy. Neuropsychiatry Neuropsychol Behav Neurol 1997; 10:243-246.

40. Wandschneider B, Thompson P, Vollmar C, Koepp M. Frontal lobe function and structure in juvenile myoclonic epilepsy: a comprehensive review of neuropsychological and imaging data. Epilepsia 2012;53:2091-2098.

41. Fraascareli Pascalicchio T, de Araujo Filho G, Helena da Silva Noffs M, et al. Neuropsychological profile of patients with juvenile myoclonic epilepsy: a controlled study of 50 patients. Epilepsy Behav 2007;10:263-267.

42. Wandschneider B, Centeno M, Vollmar C, et al. Risk-taking behavior in juvenile myoclonic epilepsy. Epilepsia 2013;54:2158-2165.

43. Bioh R, Badoe E, Klutse K, Durowaa R. Neuropsychological deficits in children with epilepsy in Ghana: a study at Korle-Bu teaching hospital. Int Neuropsychiatr Dis J 2017;10:4. 


\section{Neurology}

\section{Genetic generalized and focal epilepsy prevalence in the North American SUDEP Registry \\ Chloe Verducci, Daniel Friedman, Elizabeth Donner, et al.}

Neurology 2020;94;e1757-e1763 Published Online before print March 26, 2020

DOI 10.1212/WNL.0000000000009295

This information is current as of March 26, 2020

\section{Updated Information \&} Services

References

Citations

Subspecialty Collections

Permissions \& Licensing

Reprints including high resolution figures, can be found at: http://n.neurology.org/content/94/16/e1757.full

This article cites 42 articles, 4 of which you can access for free at: http://n.neurology.org/content/94/16/e1757.full\#ref-list-1

This article has been cited by 1 HighWire-hosted articles: http://n.neurology.org/content/94/16/e1757.full\#\#otherarticles

This article, along with others on similar topics, appears in the following collection(s):

\section{All Epilepsy/Seizures}

http://n.neurology.org/cgi/collection/all_epilepsy_seizures

Information about reproducing this article in parts (figures,tables) or in its entirety can be found online at:

http://www.neurology.org/about/about_the_journal\#permissions

Information about ordering reprints can be found online:

http://n.neurology.org/subscribers/advertise

Neurology ${ }^{\circledR}$ is the official journal of the American Academy of Neurology. Published continuously since 1951, it is now a weekly with 48 issues per year. Copyright Copyright ( 2020 The Author(s). Published by Wolters Kluwer Health, Inc. on behalf of the American Academy of Neurology.. All rights reserved. Print ISSN: 0028-3878. Online ISSN: 1526-632X.

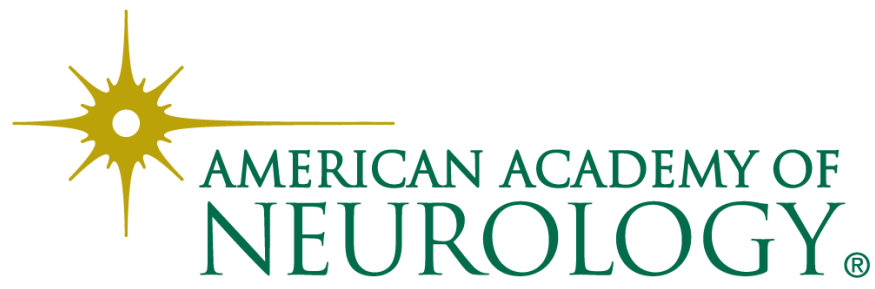

